\title{
Effect of Diameter Ratio on Loop Strength of Nylon Guts and Stainless Steel Filaments
}

\author{
By Atsuo Konda, Toshimasa Misaizu, and Sadamu Sekiguchi, Members, TMSJ \\ Faculty of Textile Science and Technology, Shinshu University, Ueda City, Nagano Prefecture
}

Based on the Journal of the Textile Machinery Society of Japan, Transactions, Vol. 30, No. 8, T139-T147 (1977)

\begin{abstract}
The loop strength of nylon guts and soft stainless steel filaments was measured and the relation between it and the load-elongation curve in simple tensile test was investigated. The results obtained are as follows:

1. The loop strength of samples used increases with increasing the diameter ratio. The degree of increase is dependent upon the tensile breaking strain of samples.

2. The following experimental equations were obtained for nylon guts:

$$
\Delta e=\varepsilon_{m o} \ldots . . .(\mathrm{a}), \quad \quad e_{m o}=1 /\left(1+\varepsilon_{r} \gamma_{d o}\right) \ldots . .(\mathrm{b})
$$

where $\Delta e$ is the decrease of strain which is defined as the difference between the tensile breaking strain and its tnesile strain at the breaking point in the loop test, $e_{m o}$ is geometric bending strain calculated from the micrographs of the hooked part, $\gamma_{d o}$ is diameter ratio, and $\varepsilon$ and $\varepsilon_{r}$ are constants independent of the diameter ratio. Therefore, the loop strength for a given diameter ratio can be estimated from both the above equations and their load-elongation curves obtained by simple tensile tests.
\end{abstract}

\section{Introduction}

In the previous paper ${ }^{[1]}$, it was pointed out that:

1) The loop strength of nylon, polyester, and soft stainless steel fibers decreases with decreasing the tensile breaking strain.

2) It is estimated by scanning-electron-microscopic observation of the rupture surface that the rupture of a nylon filament in the loop test begins at the outer side of the bending part.

3) If it is assumed that the rupture of a filament in the loop test takes place at the region where the sum of the bending strain $e_{m}$ and the tensile strain $e_{f}$ has just reached the breaking strain $e_{B}$ obtained in the simple tensile test of the filament, the decrease of breaking strain (defined as the difference between the breaking strain $e_{B}$ in the tensile test and the tensile strain $e_{f b}$ at the breaking point in the loop test) should be nearly equal to the bending strain $e_{m}\left(e_{f b} \ll 1\right)$.

(4) This bending strain $e_{m}$ decreases with decreasing $e_{B}$, and the experimental equation $\Delta e=e_{m}=B e_{B}$ is obtained. were $B$ is 0.56 for nylon 6 and 0.95 for stainless steel filaments respectively.

5) Above results being approved, the loop strength can be estimated from the simple tensile load-elongation curve, since on this curve the load at the point where strain is equal to $\left(e_{B}-\Delta e\right)$ should be one half of the loop strength.

However, the measurement of this bending strain $e_{m}$ by photo-elasticity was so difficult that the validity of the above assumption in 3 ) could not be ascertained.
In this report, firstly, the shape of the broken end and its position are surveyed microscopically to examine the propriety of the above assumption on the rupture condition in the loop test. Secondly, the loop strength of a sample hooked on other single filament of different thicknesses is measured to clarify the effect of the bending strain $e_{m}$ induced by bending. On the other hand, geometrical bending strain $e_{m o}$ at different diameter ratios (the ratio of the diameter of a filament to that of the specified sample) is calculated from photographs of the bending part just before broken. Then, the relationship between $e_{m o}$ and $e_{m}(=\Delta e)$ is investigated, and finally it is discussed whether the loop strength at different diameter ratios could be estimated from the simple tensile load-elongation curves.

Moreover, two filaments having about the same thickness but different breaking strain $e_{B}$ are hooked to investigate whether there were a rule concerning which filament of them is broken in the loop test.

\section{Definition of Terms}

Loop strength ratio: Ratio of one half of the loop strength to the simple tensile breaking strength.

Sample: Weaker filament which is subjected to the loop test, and arranged so that this filament may always break and the other filament or filaments hooked on may always remain unbroken.

Diameter ratio: Ratio of diameter $D_{02}$ of a filament to diameter $D_{01}$ of the sample. 
Decrease of strain: Difference between the breaking strain in the simple tensile test and the tensile strain $e_{f b}$ at the broken point in the loop test.

$\mathrm{N}$ gut: Nylon gut

SS filament (or SS): Soft stainless steel filament

HS filament (or HS): Hard steel filament for a fishing line.

\section{Experimental Method}

3.1 Effect of diameter ratios

\section{A. Filaments}

Commercial $\mathbf{N}$ guts, SS filaments, and HS filaments were used, the diameter of which are shown in Table 1 (a). Other properties are tabulated in Table 1 (b).

B. Combination of two filaments hooked mutually

Combination of filaments tested, and their diameter ratios are shown in Table 2.

Diameters of samples used in IB and IIB are larger than those in IA and IIA, respectively, so that the loop test may be performed at the diameter ratio smaller than unity.

Table 1. Samples used

(a) Diameters

\begin{tabular}{ll}
\hline Samples & Diameters $(\mathrm{mm})$ \\
\hline $\mathrm{N}$ (nylon) gut & $0.129,0.171,0.290,0.370$ \\
\hline $\begin{array}{l}\text { SS (Soft stainless } \\
\text { steel) filament }\end{array}$ & $0.025,0.030,0.040,0.050,0.10,0.20$ \\
\hline $\begin{array}{l}\text { HS (hard steel) } \\
\text { filament }\end{array}$ & $0.102,0.130,0.171,0.194,0.250$ \\
\hline
\end{tabular}

(b) Notation, diameters and tensile properties

\begin{tabular}{lllcc}
\hline & Notation & Diameter $(\mathrm{mm})$ & Breaking strength $\left(\mathrm{kg} / \mathrm{mm}^{2}\right)$ & Breaking strain $(\%)$ \\
\hline \multirow{2}{*}{ N gut } & N 06 & 0.129 & 76.2 & 28.5 \\
& N 1 & 0.171 & 82.4 & 29.7 \\
\hline \multirow{2}{*}{ SS filament } & S 2 & 0.025 & 85.2 & 29.4 \\
& S 2' & 0.024 & 92.8 & 9.9 \\
& S 20 & 0.20 & 76.4 & 42.0 \\
\hline
\end{tabular}

Table 2. Combination of filaments hooked and their diameter ratios

\begin{tabular}{llll}
\hline Notation & Combination & Samples & Diameter ratio \\
\hline IA & N gut-N gut & N06 & $1.00,1.33,2.25,2.87$ \\
\hline IB & N gut-HS & N1 & $0.60,0.76,1.00,1.14,1.46$ \\
\hline IIA & SS-SS & S2 & $1.00,1.20,1.60,2.00,4.00$ \\
II A & SS-SS & S2 & \\
\hline II B & SS-HS & S20 & $0.65,0.86,0.97,1.25$ \\
\hline
\end{tabular}

Table 3. Tensile properties of filaments having different tensile breaking strain

\begin{tabular}{lllll}
\hline \multirow{2}{*}{ Sample } & Notation & Tensile properties & & \\
20d Nylon & N (46) & 122.3 & Strength $\left(\mathrm{kg} / \mathrm{mm}^{2}\right)$ & Strain $(\%)$ \\
& N (24) & 137.1 & 61.2 & 46.0 \\
& E (24) & 102.2 & 77.5 & 24.6 \\
\hline \multirow{2}{*}{ 20d polyester } & E (15) & 103.6 & 63.0 & 24.5 \\
& N1 (G) & 1830 & 67.9 & 15.5 \\
\hline
\end{tabular}




\section{Simple tensile tests and loop tests}

Tensilon made by Toyo Measuring Instrument Co. was used to measure the simple tensile strength and the loop strength. The load-elongation curves were obtained at $20^{\circ} \mathrm{C}$ and $65 \% \mathrm{RH}$ at the strain rate of $100 \% / \mathrm{min}$. The distance between the upper and lower chucks was $100 \mathrm{~mm}$.

3.2 Loop tests between two filaments which have about the same thicknesses but different tensile breaking strains.

Samples having different breaking strains were made by drawing commercial polyester and nylon filaments and $\mathrm{N}$ guts in a water bath at $70^{\circ} \mathrm{C}$. The tensile breaking strengths and strains of these samples are shown in Table 3.

\section{Results and Discussion}

\subsection{Effect of diameter ratios}

A. Change of loop strengths with diameter ratios

The loop strength ratios obtained are plotted in Fig. 1 against the logarithmic diameter ratio.

In general, the loop strength ratio increases with increasing the diameter ratio. The loop strength ratio in the combination of SS filament-SS filament (IIA) rapidly increases to nearly $100 \%$ at the diameter ratio of 1.6 . On the other hand, the loop strength ratio in the combination of $\mathrm{N}$ gut- $\mathrm{N}$ gut (IA) does not increase so largely, and is $81 \%$ at the diameter ratio of 3 .

The loop strength ratio of $\mathrm{N}$ gut in the combination of $\mathrm{N}$ gut-HS filament (IB) is much larger than that in the combination of $\mathrm{N}$ gut- $\mathrm{N}$ gut at the same diameter ratio. Thus, the loop strength ratio versus diameter ratio curve for $\mathrm{N}$ gut-HS filament (IB) shifts to the left side. The loop strength ratio of SS filament in the case of SS-HS is larger at the diameter ratio of unity than that in the case of SS-SS, but nearly the same in both cases at larger diameter ratios. From these results, it is found that the loop strength ratio is remarkably affected both by the nature of the filament hooked on and the diameter ratio.

Moreover, the loop strength ratio of S2' (made by drawing SS filament S2 by $20 \%$ to give $9.9 \%$ tensile breaking strain which is lower than that of the original sample S2) is about $10 \%$ smaller than that of $\mathrm{S} 2$, as expected from the result in the previous paper ${ }^{[1]}$.

B. Shapes and positions of broken ends

It is unquestionable that the filament rupture in loop test usually takes place at the bending part. However, it serves as a good reference for investigating the breaking mechanism to ask at what position the rupture takes place. Therefore, the bending part was divided into four parts, A, B, C, and D, as shown in Fig. 2 (a), and the distribution of the rupture position was surveyed. The results are tabulated in Table 4.

For $20 \mathrm{~d}$ nylon used in the previous study ${ }^{[1]}$, those samples having tensile breaking strain of $41.3 \%$ break at A or B, and the frequency is about the same. However those having smaller breaking strain break almost at $\mathrm{A}$.
For N gut-N gut, the samples N06 having the breaking strain of $28.5 \%$ break almost at $\mathrm{A}$ when the diameter ratio is unity. As the diameter ratio increases, however, the frequency of $\mathrm{B}$ increases. When the diameter ratio is 2.87 , the frequency of $\mathrm{B}$ is very large, and that of $\mathrm{C}$ reaches to onethird.

Although this result may be caused by friction at the inside of the bending part, yet the lateral compression at the inner side seems to play an important part in this case, because the sample is wrapped by the opposite thick filament as shown in Fig. 3.

For $\mathrm{N}$ gut-HS filament, almost the same results are obtained as those for $\mathrm{N}$ gut- $\mathrm{N}$ gut. At the diameter ratio of 1.13, however, a few specimens break at $\mathrm{D}$. As the diameter ratio increases, the frequency of $\mathrm{D}$ seems to increase.

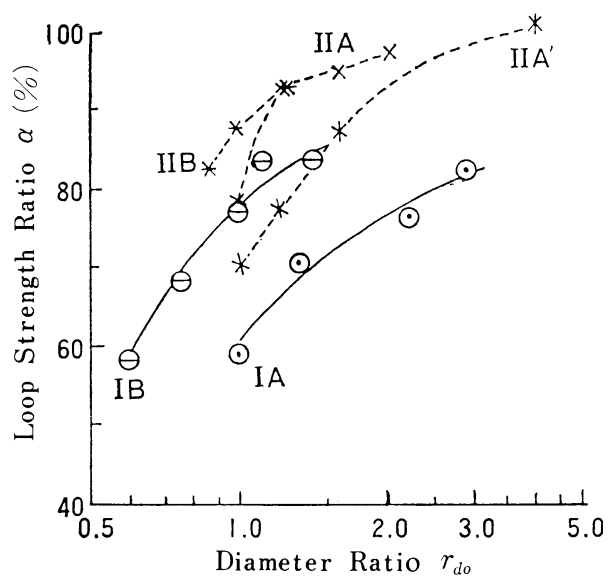

Fig. 1 Loop strength ratio versus diameter ratio

$\odot: \mathrm{N}$ gut-N gut (IA); $\times$ : SS-SS (IIA) $\ominus: \mathrm{N}$ gut-HS

(IB); $*$ : SS-HS (IIB) $*$ : SS-SS (IIA')

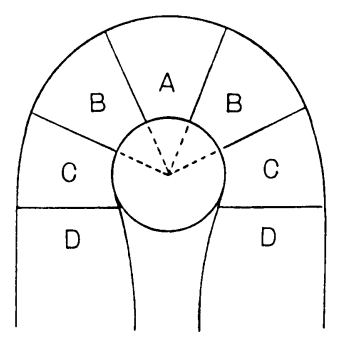

(a) Broken positions

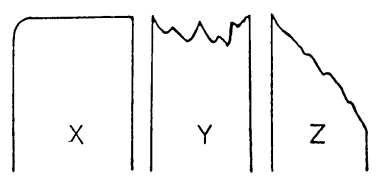

(b) Broken shapes

Fig. 2 Classification of broken shapes and broken positions 


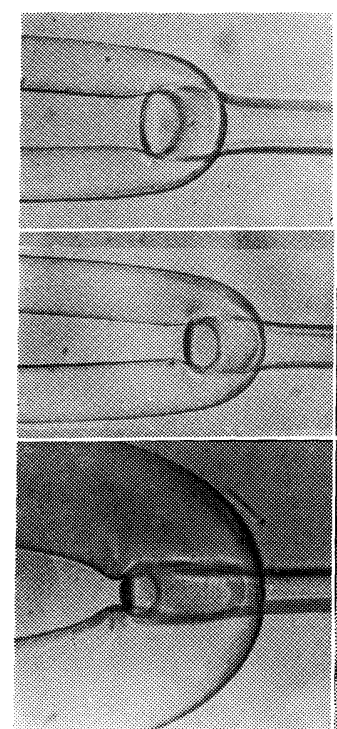

(a)

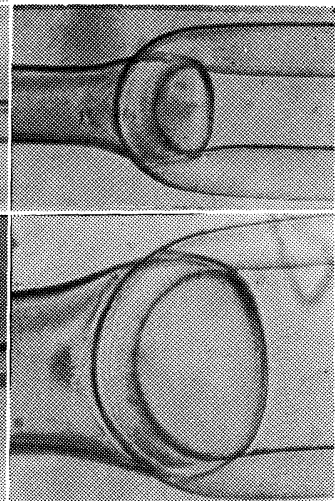

(b)
Fig. 3 Bending parts of a $\mathrm{N}$ gut(No6) just before broken in case of $\mathrm{N}$ gut- $\mathrm{N}$ gut

So, the breaking mechanism assumed in this study may be inappropriate to explain the rupture at such a large diameter ratio.

For SS filament-HS filament, the rupture always takes place at $\mathrm{A}$, at the diameter ratio of 0.86 but does not take place at $\mathrm{A}$ and mostly at $\mathrm{C}$ at the diameter ratio of 1.25 . So, it is found that the distribution of rupture position is charac- teristic for SS filament-HS filament, and the breaking mechanism of SS filament may be a little different from that of $\mathrm{N}$ gut.

When the loop strength ratio is above $90 \%$ and the scatter of tensile breaking strength is larger, some specimens of nylon or polyester filaments break near the chuck or at a straight portion (not shown in Table 4). On the contrary, SS filaments break at the bending part, even if thir loop strength ratio is above $90 \%$.

From these resuts, it is found that the assumption in the previous paper ${ }^{[1]}$ for the breaking mechanism in the loop test is appropriate for the study in this paper.

The shapes of breaking ends in the loop test are grouped into three types, $X, Y$, and $Z$ as shown in Fig. 2 (b), and their proportions are shown in Table 4 . There were no obvious correlations among them.

C. Observation of bending parts

Fig. 3 shows micrographs of samples N06 just before broken. Fig. 3 (b) was taken when the samples were rotated around the axis of stretching by $90^{\circ}$ from those in Fig. 3 (a).

The bending part of this sample N06 is wrapped in and compressed at both sides by the thicker filament. This is the reason why many samples break at $\mathrm{B}$ or $\mathrm{C}$ as mentioned above.

When N1 is hooked on HS filament of different thicknesses, the cross-section of the bending part is flattened especially at a larger diameter ratio (see Fig. 4). Youngs modulus of steel is in the order of $10^{4} \mathrm{~kg} / \mathrm{mm}^{2}$ which is much larger than that of $\mathrm{N}$ gut $\left(3-4 \times 10^{2} \mathrm{~kg} / \mathrm{mm}^{2}\right)$, and the moment of inertia

Table 4. Shapes of broken ends and their positions

\begin{tabular}{|c|c|c|c|c|c|c|c|c|}
\hline \multirow[t]{2}{*}{ Sample } & \multirow{2}{*}{$\begin{array}{l}\text { Diameter } \\
\text { ratio }\end{array}$} & \multicolumn{3}{|c|}{ Broken position $(\%)$} & \multirow[b]{2}{*}{$\mathrm{D}$} & \multicolumn{3}{|c|}{ Shape of broken end $(\%)$} \\
\hline & & A & $\mathrm{B}$ & $\mathrm{C}$ & & $\mathrm{X}$ & $\mathrm{Y}$ & $\mathrm{Z}$ \\
\hline \multirow{3}{*}{$\begin{array}{c}\text { N } 06 \\
\left(\begin{array}{c}\text { Ngut-Ngut } \\
\text { IA }\end{array}\right)\end{array}$} & 1.00 & 88.9 & 11.1 & 0 & 0 & 63.0 & 33.3 & 3.7 \\
\hline & 1.33 & 51.7 & 48.3 & 0 & 0 & 58.6 & 27.6 & 13.8 \\
\hline & 2.87 & 7.1 & 60.7 & 32.1 & 0 & 67.9 & 17.9 & 14.3 \\
\hline \multirow{4}{*}{$\begin{array}{c}\text { N 1 } \\
\left(\begin{array}{l}\text { Ngut-HS } \\
\text { IB }\end{array}\right)\end{array}$} & 0.76 & 92.3 & 7.7 & 0 & 0 & 76.9 & 5.1 & 18.0 \\
\hline & 1.00 & 94.6 & 5.4 & 0 & 0 & 83.8 & 5.4 & 10.8 \\
\hline & 1.13 & 55.3 & 38.3 & 4.3 & 2.1 & 74.5 & 8.5 & 17.0 \\
\hline & 1.46 & 38.8 & 42.9 & 10.2 & 8.1 & 71.4 & 10.2 & 18.4 \\
\hline \multirow{3}{*}{$\begin{array}{c}\text { S } 20 \\
\left(\begin{array}{c}\text { SS-HS } \\
\text { II B }\end{array}\right)\end{array}$} & 0.86 & 100.0 & 0 & 0 & 0 & & & \\
\hline & 0.97 & 40.0 & 52.5 & 7.5 & 0 & & & \\
\hline & 1.25 & 0 & 12.5 & 87.5 & 0 & & & \\
\hline \multicolumn{9}{|c|}{$\begin{array}{l}\text { Breaking } \\
\text { strain }(\%)\end{array}$} \\
\hline \multirow{4}{*}{ 20d nylon } & 1.00 & 48.1 & 51.9 & 0 & 0 & 75.0 & 17.9 & 11.2 \\
\hline & 1.00 & 79.3 & 20.7 & 0 & 0 & 55.2 & 34.5 & 10.4 \\
\hline & 1.00 & 74.3 & 20.0 & 5.7 & 0 & 48.5 & 51.4 & 0 \\
\hline & 1.00 & 89.2 & 8.1 & 2.7 & 0 & 43.2 & 56.8 & 0 \\
\hline \multirow{4}{*}{$\begin{array}{r}\text { 20d nylon } 41.3 \\
33.0 \\
24.2 \\
21.5\end{array}$} & normal & & & & & 82.4 & 5.9 & 11.8 \\
\hline & tensile & & & & & 66.7 & 25.9 & 7.4 \\
\hline & test & & & & & 78.1 & 15.5 & 6.3 \\
\hline & & & & & & 58.3 & 41.7 & 0 \\
\hline
\end{tabular}




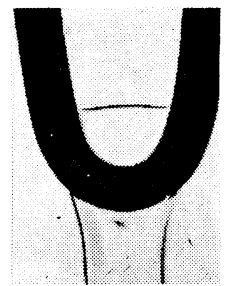

(a)

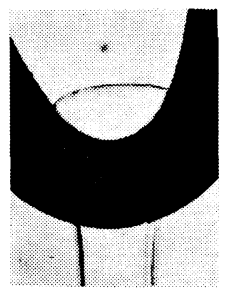

(b)

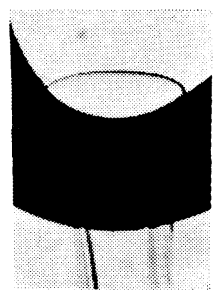

(c)
Fig. 4 Bending parts of a $\mathrm{N}$ gut(N1) just before broken in the combination of $\mathrm{N}$ gut-HS; Diameter of HS filament:

(a) 0.102 , (b) 0.194 , (c) 0.250

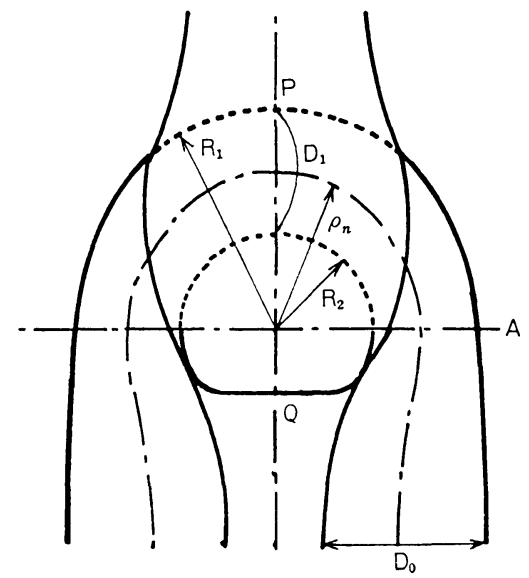

Fig. 5 Schematic diagram of the bending part in a loop tesî

of a circular-section is proportional to the fourth power of its diameter. Therefore, the flexural rigidity of HS filament rapidly increases with increasing the diameter. As a result, the curvature of the bending part of the thick HS filament is small, and so the cross-section of $\mathbf{N}$ gut is considerably flattened (the ratio of the major axis to the minor axis is about 3 for the sample in Fig. 4 (c)).

A schematic diagram of the bending part in a loop test is shown in Fig. 5. In this figure, the sample is drawn parallel with the sheet (different from its position in Fig. 4).

As mentioned in the previous paper ${ }^{[1]}$, it is difficult to decide the position of the neutral axis in the bending part. Therefore, the following equation is assumed:

$$
\rho_{n}=\left(R_{1}+R_{2}\right) / 2=D_{1} / 2+R_{2}
$$

where $\rho_{n}$ is the radius of curvature of the sample's neutral axis in the bending part. The geometrical bending strain $e_{m o}$ at outside of the bending part is defined as

$$
\begin{aligned}
e_{m o} & =D_{1} / 2 \rho_{n}=1 /\left(1+\gamma_{d}\right) \\
\gamma_{d} & =2 R_{2} / D_{1}
\end{aligned}
$$

Figs. 3 and 4 were enlarged to measure $R_{1}, R_{2}$, and $D_{1}$. $\rho_{n}$ and $e_{m o}$ calculated from eqs. (1) and (2) are plotted in Fig. 6. $\rho_{n}$ for $\mathbf{N}$ gut-HS filament is smaller than that for $\mathbf{N}$ gut-N gut. This may be because:

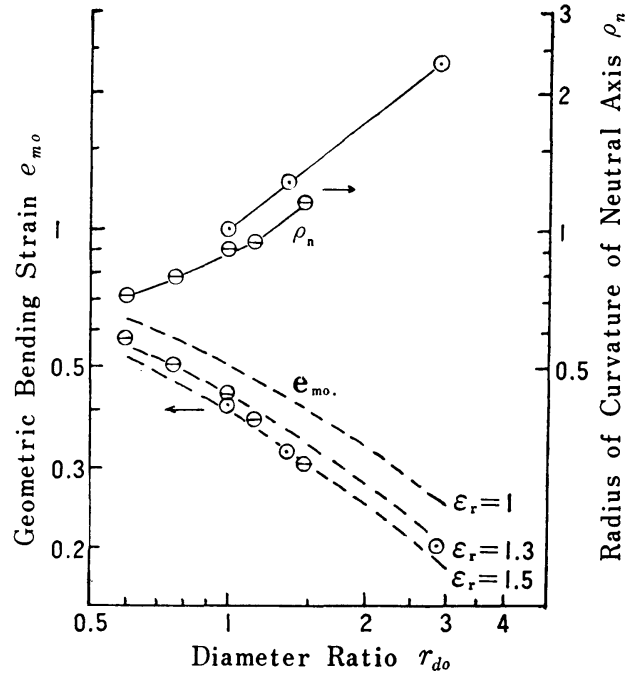

Fig. 6 Geometric bending strain, radius of curvature of neutral axis, and diameter ratio; $\odot: \mathrm{N}$ gut-N gut, $\ominus: \mathrm{N}$ gut-HS

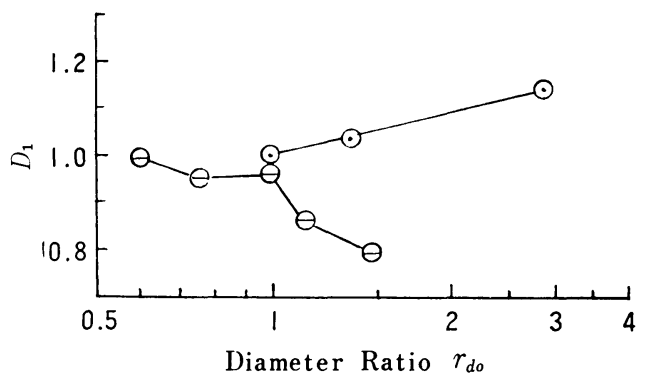

Fig. $7 \mathrm{D}_{1}$-versus diameter ratio; $\odot: \mathrm{N}$ gut-N gut, $\ominus: \mathrm{N}$ gut-HS

(1) $R_{2}$ of HS filament is nearly equal to the radius of the straight part. But $R_{2}$ of $\mathrm{N}$ gut is larger than the radius of the straight part, owing to the distortion of the bending part; (2) The cross-section of the sample in the case of $\mathrm{N}$ gut-HS filament is flattened to decrease $D_{1}$.

As the diameter ratio increases, $D_{1}$ increases in the case of $\mathrm{N}$ gut- $\mathrm{N}$ gut, but decreases in the case of $\mathrm{N}$ gut-HS filament to show smaller values than that in the former case (Fig. 7). Thus, the calculated $e_{m o}$ in Fig. 6 is about the same in both cases.

Let define $\varepsilon_{r}$ with the following equation:

$$
\gamma_{d}=\varepsilon_{r} \gamma_{d o}
$$

Then, we obtain from eq (2):

$$
\left.e_{m o}=1 / 1+\varepsilon_{r} \gamma_{d o}\right)
$$

This $\varepsilon_{r}$ is a coefficient depending upon the degree of crosssectional distortion of the bending part. Broken lines in Fig. 6 are calculated values of $e_{m o}$ for three $\varepsilon_{r}$ values 1 , 1.3 and 1.5. Although the loop strength ratio for $\mathrm{N}$ gut- $\mathrm{N}$ gut is smaller than that for $\mathrm{N}$ gut-HS filament, the values 


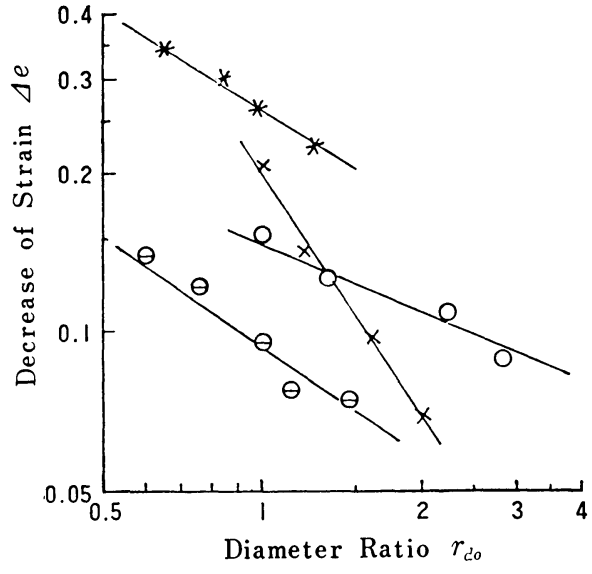

Fig. 8 Decrease of strain $\Delta e$ versus diameter ratio: $\odot: \mathrm{N}$ gut$\mathrm{N}$ gut(IA), $\ominus: \mathrm{N}$ gut-HS(IB), $\times:$ SS-SS (IIA) $*:$ SS-HS (II B)

of $e_{m o}$ in both cases are the same at the diameter ratio of unity. This may be caused by the following reasons: (1) In the case of $\mathrm{N}$ gut- $\mathrm{N}$ gut, the specified sample is wrapped in and compressed at both sides by the other filament. (2) In the case of $\mathrm{N}$ gut-HS filament, the neutral bending axis of $\mathrm{N}$ gut may be shifted towards the outside owing to the large cross-sectional distortion of $\mathrm{N}$ gut as shown in Fig. 4. As a result, the bending strain $e_{m}$ is much decreased in comparison with the case of $\mathrm{N}$ gut- $\mathrm{N}$ gut.

D. Decrease of strain

The decrease of strain, $\Delta e$, defined above was obtained from the simple tensile load-elongation curve by the same method as in the previous paper ${ }^{[1]}$, and is plotted in Fig. 8 against the diameter ratio on a log-log scale.

As the diameter ratio increases, $\Delta e$ for SS filament-SS filament rapidly decreases, and the loop strength ratio increases to nearly $100 \%$. For $\mathrm{N}$ gut-N gut, however, $\Delta e$ decreases more gradually with increasing the diameter ratio, and the loop strength ratio does not increase so much. $\Delta e$ for $\mathrm{N}$ gutHS filament is smaller than that for $\mathrm{N}$ gut- $\mathrm{N}$ gut, and the loop strength ratio for the former is larger than that for the latter. $\Delta e$ for SS filament-HS filament is larger than $\Delta e$ for SS filament-SS filament, since the tensile breaking strain of the former sample is larger than that of the latter (refer to the previous paper).

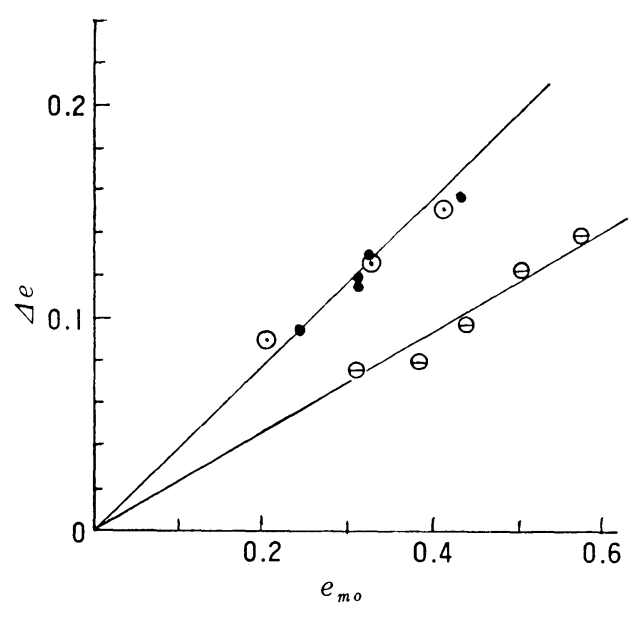

Fig. 9 Decrease of strain $\Delta e$ versus geometric bending strain $e_{m o}$ $\odot: \mathrm{N}$ gut-N gut, $\ominus: \mathrm{N}$ gut-HS

The relation between $\Delta e$ and $e_{m o}$ for $\mathrm{N}$ gut- $\mathrm{N}$ gut and $\mathrm{N}$ gut-HS filament is approximately linear as shown in Fig. 9, and can be expressed by:

$$
\Delta e=\varepsilon e_{m o}
$$

where $\varepsilon$ is a constant, and is 0.394 for $\mathrm{N}$ gut- $\mathrm{N}$ gut and 0.234 for $\mathrm{N}$ gut-HS filament. From these results, it is found that the geometric bending strain $e_{m o}$ is an important factor of $\Delta e$ or $\alpha$. Moreover, it is reasonable to assume that $e_{m o}$ decreases at a constant rate $\varepsilon$ in case of $\mathbf{N}$ gut- $\mathrm{N}$ gut or $\mathrm{N}$ gut-HS filament, and the real bending strain $e_{m}$ becomes $\varepsilon e_{m o}$. From this assumption, the effect of the diameter ratio on the loop strength is quantitatibly accounted for.

If the load $f$ in the simple extension of $\mathrm{N}$ gut is a function of the tensile strain $e$, and represented by

$$
f=F(e),
$$

the loop strength $f_{L}$ and the loop strength ratio $\alpha$ of $\mathrm{N}$ gut are expressed as follows:

$$
\begin{aligned}
& f_{L}=2 F\left(e_{B}-\Delta e\right) \ldots \ldots \ldots \ldots \ldots \ldots \\
& \alpha=f_{L} / 2 f_{B}=F\left(e_{B}-\Delta e\right) / F\left(e_{B}\right) \\
& \Delta e=\varepsilon e_{m o}=\varepsilon /\left(1+\varepsilon_{r} \gamma_{d o}\right) \ldots \ldots
\end{aligned}
$$

On the other hand, as found from the results in Fig. 8, the following experimental equation holds for all the samples used:

Table 5. Values of $\mathrm{c}$ and $\mathrm{n}$ in eq. (10)

\begin{tabular}{lllll}
\hline Notation & Samples & Combination & C & $\mathrm{n}$ \\
\hline I A & N 06 & N gut-N gut & 0.147 & 0.434 \\
I B & N 1 & N gut-HS & 0.095 & 0.693 \\
II A & S 2 & SS-SS & 0.200 & 0.153 \\
II B & S 20 & SS-HS & 0.262 & 0.647 \\
\hline
\end{tabular}




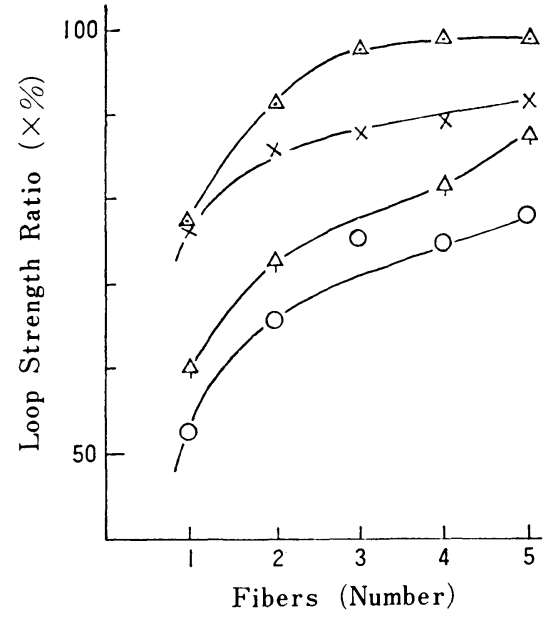

Fig. 10 Loop strength ratio versus numbers of filaments hooked on; $\odot$ : N gut (N1), $\quad \times:$ SS filament (S2) $\triangle$ : Polyes ter $\mathrm{E}(24), \triangle$ : Polyester $\mathrm{E}(14)$

$$
\Delta e=C \gamma d o^{-n} \text {....... }
$$

where $C$ and $n$ are constants shown in Table 5 .

Althought the physical meaning of $C$ and $n$ is ambiguous, the loop strength for different diameter ratios can be obtained from eq. (7) or the simple tensile load-elongation curve by using $\Delta e$ calculated from eq. (10). $C$ represents $\Delta e$ at the diameter ratio of unity, and takes a large value for large breaking strain $e_{B}$ and/or $\varepsilon$ in eq. (5)

E. Loop strength when hooked on numbers of filaments

Fig. 10 shows the loop strength ratio hooked on a bundle composed of numbers of filaments in place of a single filament. The characteristics are summarized in Table 3, excepting the sample $\mathrm{E}(14)$ which was made by drawing the sample $\mathrm{E}(24)$.

The loop strength ratio increases with incresasing the number of other filaments, especially with the change from 1 to 2 .

Micrographs of the bending part of $\mathrm{N}$ gut just before broken are shown in Fig. 11. $e_{m o}$ calculated from them is plotted with dot in Fig. 9. Almost the same relation is obtained, between $\Delta e$ and $e_{m o}$ as that for $\mathrm{N}$ gut- $\mathrm{N}$ gut (IA), because the cross-section of bundled filaments is so distorted that the sample hooked on the bundle is smoothly bent as shown in Fig. 11.

Fig. 12 shows the simple tensile load-elongation curves of various samples which are normalized in order that their breaking load and elongation may be unity. The loop strength ratio of the sample $\mathrm{N} 1$ at the diameter ratio of unity is equal to the load value at the elongation of $0.44(=1-$ $\left.\Delta e / e_{B}\right)$ on this normalized curve, because $\Delta e / e_{B}$ obtained for $\mathrm{N} 1$ in the previous paper ${ }^{[1]}$ is 0.56 , which is equal to the decrease of the normalized strain. If it is assumed that $\Delta e / e_{B}$
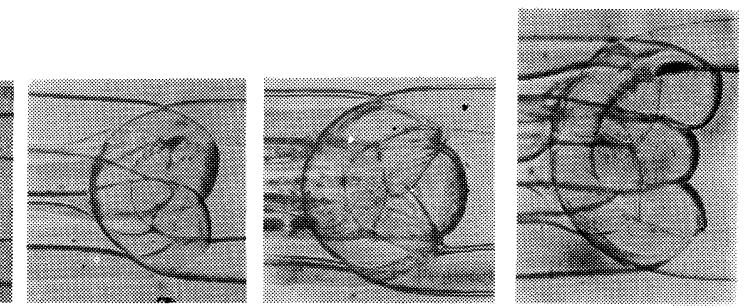

Fig. 11 Bending parts of a $\mathrm{N}$ gut hooked on numbers of $\mathrm{N}$ guts and stretched until just before broken

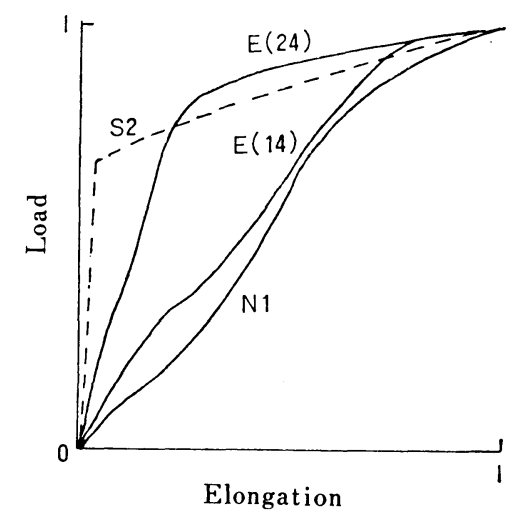

Fig. 12 Normalized load-elongation cnrves of various samples

for polyester samples is about the same as that for nylon gut $\mathrm{N} 1$, the loop strength ratio of $\mathrm{E}(14)$ and $\mathrm{E}(24)$ must be larger than that of $\mathrm{N} 1$, and that of $\mathrm{E}(24)$ larger than that of $\mathrm{E}(14)$, as found from Fig. 12. Furthermore, as the diameter ratio increases, $\Delta e$ decreases so that the point $\left(1-\Delta e / e_{B}\right)$ on the normalized load-elongation curve shifts to the right, and thus the loop strength ratio must increase along the curve in Fig. 12. Actually, each loop strength ratio of samples in Fig. 10 increases with increasing diameter ratio, as estimated from Fig. 12.

4.2 Loop test of various samples

Results of the loop test of various samples are tabulated in Table 6. In this case two filaments hooked each other have about the same thickness but different breaking strains (See Table 3). Table 6 represents the proportion that the sample in the left column is broken with the filaments shown in the upper column. Sample E(15) having the smallest breaking strain is always broken at a large percentage not less than $80 \%$. Although there are cases where two filaments hooked each other break at the same time, these specimens are excluded from the percentage calculation. Sample N1 (G) is always broken with sample N1 (C) which has larger breaking strain than N1 (G), though the breaking strength of N1 (G) is larger than that N1 (C). This sample N1 (C) is sold as a gut having better quality and larger loop strength ratio than 
(a)

Table 6. Proportion of broken samples in the loop test

\begin{tabular}{lccccc}
\hline Samples observed & \multicolumn{5}{c}{ Opposite filaments } \\
\cline { 2 - 6 } & N (46) & N (24) & E (24) & E (15) \\
\hline Nylon & N (46) & - & 7 & 10 & 0 \\
\hline Nylon & N (24) & 93 & - & 44 & 10 \\
\hline Polylster & E (24) & 90 & 56 & - & 20 \\
\hline polylster & E (15) & 100 & 90 & 80 & - \\
\hline
\end{tabular}

(b)

\begin{tabular}{lllll}
\hline Samples & \multicolumn{3}{l}{ Opposite filaments } \\
\cline { 3 - 5 } & & N1 $(\mathrm{C})$ & N1 $(\mathrm{G})$ & N1 $\left(\mathrm{C}^{\prime}\right)$ \\
\hline N gut & N1 $(\mathrm{C})$ & - & 0 & 35 \\
\hline N gut & N1 $(G)$ & 100 & - & 57 \\
\hline N gut & N1 $\left(C^{\prime}\right)^{*}$ & 65 & 43 & -
\end{tabular}

* N1 ( $\left.\mathrm{C}^{\prime}\right)$ was made by drawing N1 (C) to let it have about the same breaking strain of $26.0 \%$ as that of $\mathrm{N} 1(\mathrm{G})$.

N1 (G). However, N1 $\left(C^{\prime}\right)$ which is prepared by drawing N1 (C) to give about the same breaking strain as N1 (G) is broken at a large percentage ( $43 \%$ ) with N1 (G). It is concluded from these results that the sample of smaller breaking strain is broken at larger percentage in this experiment. Although the loop test between polyester and nylon filaments should be carried out to insist this conclusion, such samples as having the same thickness could not be prepared.

On the other hand, the sample heat-treated above $160^{\circ} \mathrm{C}$ for nylon or above $200^{\circ} \mathrm{C}$ for polyester has a tendency to have smaller loop strength ratio than the untreated sample. Hence, it must be borne in mind that the loop strength is affected by heat-treatment besides its tensile breaking strain. Among samples shrunk to different lengths by heattreatment, however, the larger their breaking strain is, the larger their loop strength ratio is.

\section{Summary}

A. The loop strength of $\mathrm{N}$ guts and SS filaments was measured, and the relationship between the diameter ratio and the geometrical bending strain, decrease of strain, were investigated.

1. The loop strength of samples used increases with increas- ing the diameter ratio. The degree of increase is dependen upon the simple tensile breaking strain of samples.

2. The loop strength is affected by the shape of distorted cross-section and the curvature of bending part of the opposite filament hooked on.

3. The following experimental equations were obtained for sample N1:

$$
\Delta e=\varepsilon e_{m o} \quad \ldots . . .(5) ; \quad e_{m o}=1 /\left(1+\varepsilon_{r} \gamma_{d o}\right)
$$

$\varepsilon$ is 0.394 for $\mathbf{N}$ gut and 0.234 , for $\mathbf{N}$ gut-HS filament, and is independent of diameter ratio.

So, $e_{m o}$ is an important factor for the loop strength. The loop Strength $f_{L}$ for a given diameter ratio can be estimated from both the above equations and their load-elongation curves of simple tensile test.

4. The following assumption proposed in the previous paper is concluded to be appropriate: a fiber in loop test begins to rupture at the region where the sum of bending and tensile strains has just reached the tensile breaking strain of the fiber.

B. In the loop test between two different filaments, which have about the same thicknesses but different tensile breaking strains, the specimen having the smaller tensile breaking strain is apt to be broken. This is because the loop strength of the samples used in this experiment decreases with decreasing the tensile breaking strain.

C. When a single gut of $\mathrm{N} 1$ is hooked on the bundle composed of different numbers of $\mathrm{N} 1$, it is bent smoothly along the surface of the bundle. Therefore the relation between $e_{m_{o}}$ and $\Delta e$ in this case is nearly the same as that when hooked on a single $\mathrm{N}$ gut of different thicknesses.

Furthermore, the loop strength ratios of polyester and SS filaments, each of which is hooked on its bundle, also increases with increasing the numbers of other hooked filaments, as estimated from their load-elongation curves.

The authors wish to thank Miss S. Kurashima for carrying out the loop test with us.

\section{Literature Cited}

[1] A. Konda, S. Sekiguchi, T. Misaizu, and K. Shirakashi; J. Text Mach. Soc. Japan, 27, T-127 (1974)

[2] A. Konda, H. Ishikawa, and K. Shirakashi; J. Text. Mach Soc. Japan, 25, T-65 (1972) 